\section{OP0091 TREATMENT OF OCULAR SARCOIDOSIS. STUDY OF 65 PATIENTS OF A SERIES OF 384 PATIENTS FROM A SINGLE UNIVERSITY HOSPITAL}

C. Álvarez-Reguera ${ }^{1}$, J. J. Gaitán-Valdizán ${ }^{2}$, R. Fernández-Ramón ${ }^{2}$ R. Demetrio-Pablo², J. L. Martín-Varillas ${ }^{3}$, L. Sanchez-Bilbao ${ }^{1}$, D. MartínezLópez ${ }^{1}$, I. González-Mazón ${ }^{1}$, M. Á. González-Gay ${ }^{1}$, R. Blanco ${ }^{1} .{ }^{1}$ Hospital Universitario Marqués de Valdecilla, Rheumatology, Santander, Spain; ${ }^{2}$ Hospital Universitario Marqués de Valdecilla, Ophthalmology, Santander, Spain; ${ }^{3}$ Hospital de Sierrallana, Rheumatology, Torrelavega, Spain

Background: Ocular involvement is a relatively frequent and potentially severe complication of sarcoidosis. Oral corticosteroids (OCS) are the first-line treatment. Conventional immunosuppressive agents (cIS) and biological therapy (BT) can be used in refractory cases (1-5).

Objectives: To evaluate the treatment and visual outcomes of a cohort of patients diagnosed with ocular sarcoidosis.

Methods: Study of a large cohort $(n=384)$ of all consecutive patients diagnosed with sarcoidosis from January 1, 1999 to December 31, 2019 at a single University Hospital. Finally, 344 patients were included according the ATS/ERS/ WASOG criteria (Eur Respir J. 1999; 14:735-7). Different ocular manifestations and the following systemic treatments were assessed: a) OCS, b) cIS), c) monoclonal TNF inhibitors, d) Etanercept (ETN), e) Tocilizumab (TCZ). Best Corrected Visual Acuity (BCVA) according to different systemic treatments was compared at diagnosis and after one year of follow-up (Kruskall Wallis test).

Results: 344 patients were reviewed. From these, $65(18.9 \%)$ presented ocular manifestations as uveitis $(83.1 \%)$, orbital lesions $(7.7 \%)$, retinal vasculitis $(6.2 \%)$, dry eye $(6.2 \%)$ and scleritis $(1.5 \%)$. All of them received systemic treatment. BT was particularly used in patients with retinal vasculitis $(100 \%)$, panuveitis $(75 \%)$ and orbital lesions (40\%). Systemic treatment and BCVA outcome according to

Table 1. Median BCVA at onset and after one year according to ocular manifestations and type of systemic therapy.

\begin{tabular}{|c|c|c|c|c|c|c|c|c|}
\hline $\begin{array}{l}\text { Type of ocular } \\
\text { affectation }\end{array}$ & $n(\%)$ & $\begin{array}{c}\text { Median } \\
\text { BCVA } \\
\text { at onset } \\
\text { [IQR] }\end{array}$ & $\begin{array}{c}\text { Median } \\
\text { BCVA } \\
\text { after } 1 \text { year } \\
\text { [IQR] }\end{array}$ & $\begin{array}{l}\text { OCS } \\
n(\%)\end{array}$ & $\begin{array}{l}c / S \\
n(\%)\end{array}$ & $\begin{array}{c}\text { monoclonal } \\
\text { TNFi } \\
n(\%)\end{array}$ & ETN & $\begin{array}{l}\text { TCZ } \\
n(\%)\end{array}$ \\
\hline Uveitis & $\begin{array}{c}54 \\
(83.1)\end{array}$ & $\begin{array}{c}0.6 \\
{[0.3-0.8]}\end{array}$ & $\begin{array}{c}0.9 \\
{[0.6-1]}\end{array}$ & $\begin{array}{c}44 \\
(81.5)\end{array}$ & $\begin{array}{c}29 \\
(53.7)\end{array}$ & $\begin{array}{c}16 \\
(29.6)\end{array}$ & $\begin{array}{c}3 \\
(5.5)\end{array}$ & $\begin{array}{c}3 \\
(5.5)\end{array}$ \\
\hline -Anterior & $\begin{array}{c}31 \\
(47.7)\end{array}$ & $\begin{array}{c}0.7 \\
{[0.3-0.8]}\end{array}$ & $\begin{array}{c}0.8 \\
{[0.5-1]}\end{array}$ & $\begin{array}{c}22 \\
(70.9)\end{array}$ & $\begin{array}{c}12 \\
(38.7)\end{array}$ & $\begin{array}{c}2 \\
(6.5)\end{array}$ & $\begin{array}{c}2 \\
(6.5)\end{array}$ & 0 \\
\hline -Intermediate & $\begin{array}{c}2 \\
(3.1)\end{array}$ & 0.5 & 0.7 & $\begin{array}{c}2 \\
(100)\end{array}$ & $\begin{array}{c}1 \\
(50)\end{array}$ & $\begin{array}{c}1 \\
(50)\end{array}$ & $\begin{array}{c}1 \\
(50)\end{array}$ & $\begin{array}{c}1 \\
(50)\end{array}$ \\
\hline -Posterior & $\begin{array}{c}5 \\
(5.2)\end{array}$ & $\begin{array}{c}0.5 \\
{[0.1-0.9]}\end{array}$ & $\begin{array}{c}0.9 \\
{[0.9-1]}\end{array}$ & $\begin{array}{c}4 \\
(80)\end{array}$ & $\begin{array}{c}4 \\
(80)\end{array}$ & $\begin{array}{c}3 \\
(60)\end{array}$ & 0 & 0 \\
\hline -Panuveitis & $\begin{array}{c}16 \\
(24.6)\end{array}$ & $\begin{array}{c}0.4 \\
{[0.2-0.7]}\end{array}$ & $\begin{array}{c}0.9 \\
{[0.5-1]}\end{array}$ & $\begin{array}{c}16 \\
(100)\end{array}$ & $\begin{array}{c}12 \\
(75)\end{array}$ & $\begin{array}{c}10 \\
(62.5)\end{array}$ & 0 & $\begin{array}{c}2 \\
(12.5)\end{array}$ \\
\hline $\begin{array}{l}\text { Orbital } \\
\text { lesions }\end{array}$ & $\begin{array}{c}5 \\
(7.7)\end{array}$ & $\begin{array}{c}0.5 \\
{[0.1-0.6]}\end{array}$ & $\begin{array}{c}1 \\
{[0.1-1]}\end{array}$ & $\begin{array}{c}4 \\
(80)\end{array}$ & $\begin{array}{c}2 \\
(40)\end{array}$ & $\begin{array}{c}2 \\
(40)\end{array}$ & 0 & $\begin{array}{c}1 \\
(20)\end{array}$ \\
\hline $\begin{array}{l}\text { Retinal } \\
\quad \text { vasculitis }\end{array}$ & $\begin{array}{c}4 \\
(6.2)\end{array}$ & $\begin{array}{c}0.6 \\
{[0.5-0.8]}\end{array}$ & $\begin{array}{c}1 \\
{[0.6-1]}\end{array}$ & $\begin{array}{c}4 \\
(100)\end{array}$ & $\begin{array}{c}4 \\
(100)\end{array}$ & $\begin{array}{c}1 \\
(25)\end{array}$ & 0 & $\begin{array}{c}1 \\
(25)\end{array}$ \\
\hline $\begin{array}{l}\text { Dry eye } \\
\text { Scleritis }\end{array}$ & $\begin{array}{l}4(6.2) \\
1(1.5)\end{array}$ & $\begin{array}{l}1 \\
1\end{array}$ & $\begin{array}{c}0.9 \\
1\end{array}$ & $\begin{array}{l}2(50) \\
1(100)\end{array}$ & $\begin{array}{c}1(25) \\
0\end{array}$ & $\begin{array}{l}0 \\
0\end{array}$ & $\begin{array}{l}0 \\
0\end{array}$ & $\begin{array}{l}0 \\
0\end{array}$ \\
\hline
\end{tabular}

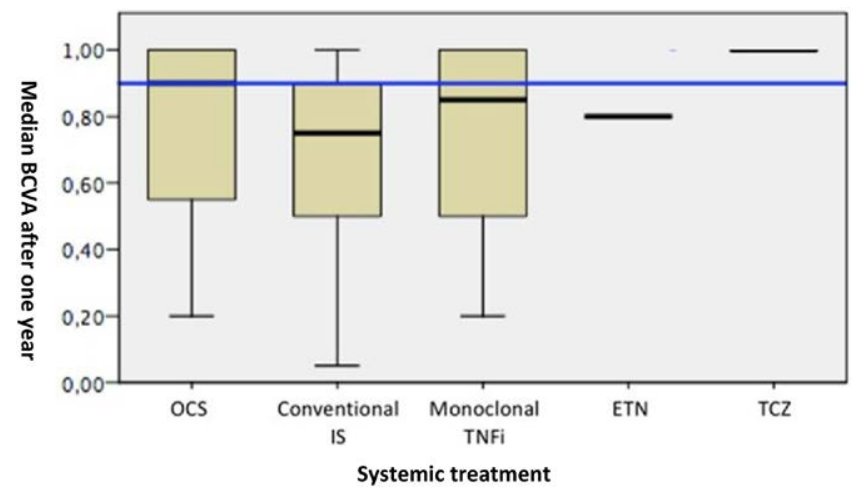

Figure 1. Median [25,75 IQR] BVCA after one year follow up according to type of systemic treatment in sarcoid uveitis.Median BCVA after one year Systemic treatmentAbbreviations: BCVA: Best Corrected Visual Acuity; OCS: Oral Corticosteroids; Conventional IS: Conventional immunosupressants; Monoclonal TNFi: monoclonal tumour necrosis factor inhibitors; ETN Etanercept; TCZ: Tocilizumab. ocular manifestations are shown in table. Median BCVA at onset and after one year was 0.6 [interquartile range (IQR) $0.3-0.8$ ] and 0.9 [0.6-1], respectively. No statistically significant differences were observed between systemic treatments in BCVA of patients with uveitis after 1 year of follow-up (Figure).

Conclusion: Panuveitis, intermediate uveitis and orbital lesions, require a more aggressive treatment than other manifestations of ocular sarcoidosis. In uveitis, an important improvement in BCVA after 1 year of follow-up was observed regardless of the type of treatment used.

REFERENCES:

[1] Riancho-Zarrabeitia L, et al. Semin Arthritis Rheum 2015;45(3):361-8. PMID: 26092330

[2] Riancho-Zarrabeitia L et al. Clin Exp Rheumatol 2014;32(2):275-84. PMID: 24321604

[3] Vegas-Revenga N, et al. Am J Ophthalmol 2019; 200:85-94. PMID: 30660771

[4] Cordero-Coma et al. Mediators Inflamm 2014; 2014:717598. PMID: 24976689

[5] Calvo-Río V, et al. Clin Exp Rheumatol 2014; 32 (4 Suppl 84): S54-7. PMID: 25005576

Disclosure of Interests: Carmen Álvarez-Reguera: None declared, Jorge Javier Gaitán-Valdizán: None declared, Raúl Fernández-Ramón: None declared, Rosalía Demetrio-Pablo: None declared, José Luis Martín-Varillas: None declared, Lara Sanchez-Bilbao: None declared, David Martínez-López: None declared, Iñigo González-Mazón: None declared, Miguel Á. GonzálezGay Speakers bureau: Abbvie, Pfizer, Roche, Sanofi and MSD., Grant/research support from: Abbvie, MSD, Janssen and Roche., Ricardo Blanco Speakers bureau: Abbvie, Pfizer, Roche, Bristol-Myers, Janssen, Lilly and MSD., Grant/ research support from: Abbvie, MSD and Roche.

\section{DOI: 10.1136/annrheumdis-2021-eular.1896}

\section{OP0092 LONG-TERM SAFETY AND EFFECTIVENESS OF CANAKINUMAB IN CRYOPYRIN-ASSOCIATED PERIODIC SYNDROMES (CAPS) - 30-MONTH DATA FROM THE RELIANCE REGISTRY}

J. B. Kuemmerle-Deschner ${ }^{1}$, B. Kortus-Goetze ${ }^{2}$, P. Oommen ${ }^{3}$, A. Janda ${ }^{4}$, J. Rech ${ }^{5}$, T. Kallinich ${ }^{6}$, F. Weller-Heinemann ${ }^{7}$, G. Horneff ${ }^{8}$, I. Foeldvari ${ }^{9}$, C. Schuetz ${ }^{10}$, M. Borte ${ }^{11}$, A. Braner ${ }^{12}$, J. Weber-Arden $^{13}$, N. Blank ${ }^{14}$.

${ }^{1}$ University Hospital Tuebingen, Department of Pediatrics, Division of Pediatric Rheumatology, Tuebingen, Germany; ${ }^{2}$ University of Marburg, Division of Nephrology, Marburg, Germany; ${ }^{3}$ Heinrich-Heine-University Düsseldorf, Clinic of Pediatric Hematology, Oncology and Clinical Immunology, Duesseldorf, Germany; ${ }^{4}$ University Hospital UIm, Department of Pediatrics, Ulm, Germany; ${ }^{5}$ Friedrich-Alexander University (FAU) Erlangen-Nürnberg and Universitätsklinikum Erlangen, Department of Internal Medicine 3 Rheumatology and Immunology, Erlangen, Germany; ${ }^{6}$ Charité University Medicine Berlin, Department of Pediatrics, Division of Pulmonology, Immunology and Critical Care Medicine, Berlin, Germany; ${ }^{7}$ Klinikum BremenMitte, Prof. Hess Kinderklinik, Bremen, Germany; ${ }^{8}$ Asklepios Clinic Sankt Augustin, Asklepios Clinic Sankt Augustin, Sankt Augustin, Germany; ${ }^{9}$ Centre for Pediatric and Adolescence Rheumatology, Centre for Pediatric and Adolescence Rheumatology, Hamburg, Germany; ${ }^{10}$ Medizinische Fakultät Carl Gustav Carus, Technische Universität Dresden, Pediatrics, Dresden, Germany; ${ }^{11}$ Hospital St. Georg g GmbH Leipzig, ImmunoDeficiencyCenter Leipzig (IDCL), Leipzig, Germany; ${ }^{12}$ University Hospital Frankfurt, Department of Medicine II, Rheumatology, Frankfurt, Germany; ${ }^{13}$ Novartis Pharma GmbH, Novartis Pharma $\mathrm{GmbH}$, Nuernberg, Germany; ${ }^{14}$ University Hospital Heidelberg, Rheumatology, Heidelberg, Germany

Background: In clinical trials as well as in real-life, the IL-1ß inhibitor canakinumab leads to rapid remission of symptoms in the treatment of CAPS, a monogenic autoinflammatory disease with severe systemic and organ inflammation. Objectives: The RELIANCE registry is designed to explore long-term safety and effectiveness of canakinumab under routine clinical practice conditions in pediatric ( $\geq 2$ years) and adult patients with CAPS, including Muckle-Wells syndrome (MWS), familial cold autoinflammatory syndrome (FCAS), and neonatal onset multisystem inflammatory disease (NOMID)/chronic infantile neurological cutaneous and articular syndrome (CINCA).

Methods: This prospective, non-interventional, observational study with a 3-year follow-up enrolls patients with clinically confirmed diagnoses of CAPS routinely receiving canakinumab. In 6-monthly visits, clinical data, physician assessments and patient-reported outcomes are evaluated starting at baseline with last update at 30 months of follow-up in the total cohort including the cohort with severe CAPS subtypes (NOMID/CINCA).

Results: 91 CAPS patients (50\% female; 14 [15\%] NOMID/CINCA subtypes) were enrolled by December 2020 (Table 1). At baseline, median age was 20.5 years and median duration of prior canakinumab treatment was 6 years. 11 drug related severe adverse events per 100 patient years were reported. $68 \%$ of patients 